\title{
Neural substrates of Motor Learning Strategies in Stroke
}

Ashu Bhasin', Neha Kuthiala', M V Padma Srivastava' and Senthil Kumaran ${ }^{2 *}$

*Correspondence: senthilssk@yahoo.com

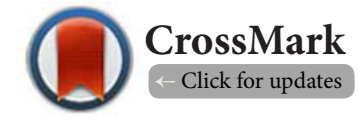

${ }^{1}$ Head, Department of Neurology, AllMS, New Delhi.

${ }^{2}$ Department of NMR, AllMS, New Delhi.

\begin{abstract}
Stroke neuro rehabilitation rely on human and animal studies about learning and adaptation. The physiological principles underlying them are based on experience dependent neuronal plasticity. The paper will revisit different motor learning strategies which can be administered in stroke (acute \& chronic). This summary also encompasses various neurorestorative interventions like CIMT, mirror therapy, VR, haptics, brain stimulation, task-oriented training, massed and repetitive practice experimented for stroke with most robust reviews and recent trials. We also expand this review with our experience on both acute and chronic stroke recovery patterns amalgamated with functional imaging.
\end{abstract}

Keywords: Functional imaging, neural control, stroke, motor rehabilitation

\section{Introduction}

Our introductory message for this manuscript commences with the famous quote of biogenesis "Ontogeny recapitulates phylogeny" coined by the eminent scientist Earnst Hackel. This enroutesus to understand that evolution of human body is believed to be on phylogenyi.e, 'evolutionary lineage of species', and ontogeny which 'purports the progression of organisms' during the embryonic development [1].

\section{Review}

\section{CNS injury, learning and neurorestoration}

According to the theory of Neural Darwinism [2], the nervous system is capable of developing synaptic connections, from one nerve cell to another, which are strengthened or weakened depending on environmental stimuli, resulting in a huge number of repertoires of variance $[\mathbf{3}, \mathbf{4}]$ ". This adaptation of human body is a continuous process which is observed as a learning process after central nervous system (CNS) injury. It, in a layman notion is teaching 'an old brain new tricks of adaptation' and learning. In the history of stroke recovery and evolution, maps are shaped during early life and remain quite stable in adulthood. Interestingly, they can change in the adult spontaneously after brain injury like stroke or by experience dependent plasticity (such as after an intensive training). Stroke induces production of various factors of neural regeneration, sprouting and plasticity, such as myelin components (Nogo-A, myelin-associated glycoprotein), and guidance molecules (ephrins, semaphorins, netrins)which aid in neuro functional recovery post stroke [5].

\section{Motor relearning and its neural connections [6,7]} Literature augments many theories describing how multiple systems come together to produce a functional movement. The first theory is the motor program theory which is based on central control of movement instructions and the second is based on dynamic self-organization of multiple sub systems with a meaningful goal.

Another theory was Schmidt's 'schema theory' where only abstract forms of movements can be stored in memory through generalized motor programs (GMPs). Motor learning can be classified into two main categories: (i) motor sequence learning that is related to the acquisition of motor skills, and (ii) motor adaptation that is capacity to compensate for environmental changes.

As explained by Newell \&Verhoeven 2015 [8] that learning 
Bhasin et al., Physical Therapy and Rehabilitation 2021,

and relearning principles may involve some different neural pathways yet there are some common set of motor dynamics in every human brain which work towards a movement. Some of the patterns are preserved, new patterns are learned and some old are relearned. The inherent individual differences of humans and environments being fed are also one of the major contributors for recovery after stroke apart from the stroke topography. Motor priming is a well known form of implicit learning that enhances efficacy of function post stroke.

\section{Motor learning and neural rehabilitation}

The techniques of neurorehabilitation by and large, unanimously are based on motor learning processes have been classified into three major stages; i) early motor learning is also called the cognitive or verbal-motor stage, and is characterizedby considerable improvement of a novel task, but inconsistencies between movements, ii) during the associative, the movements are more consistent; iii) during the autonomousst age, learners automatically perform the task, and may even do dual tasking during one task's execution $[9,10]$.

Skill acquisition has been defined as "a set of processes associated with practice leading to relatively permanent changes in the capability for responding" $[11,12]$. These motor skills are learned through practice from infancy to adults.In a previous meta-analysis by Maier et al 2019 [5], massed \& structured practice, task-specific, variable practice, implicit and explicit learning, multisensory stimulation, knowledge of results \& performance (KP KR), movement representation, recruitment $\&$ focusing and promotion of the use of the affected limb, are some of the principles for neurorehabilitation based on the concept of learning.

Meta-analyses suggest that patients with stroke can learn implicitly with their unaffected side $(95 \% \mathrm{CI}[45.1$ to 92.9$]$, $\mathrm{p}<0.0001)$, but not with their affected side $[95 \% \mathrm{Cl}(-0.45$ to $0.25), p=0.56$ ] but overall threshold did not reach a statistical significance [13]. Motor recovery after stroke always fall short of $100 \%$ in kinematics and kinetics there by leading to substitutory and compensatory strategies being learned by the injured brain for simple and complex activities. It also depends on task (activities of daily living or complex tasks or skillful tasks) and the patient's occupation (athlete, an archer's skill acquisition will be different from a desk employee). Most of the motor skills require implicit learning unless the task is new $[14,15]$.

After spawning through research articles, we found various neurorestorative interventions being experimented on stroke subjects like CIMT, mirror therapy (MT), (Virtual Reality), VR, Haptics, task oriented training, massed and repetitive practice [16].

\section{Constraint Induced movement therapy (CIMT)}

The original form of CIMT consists of intensive practice of the paretic upper limb aimed at enhancing task-specific use for up to 6 hours a day for 2 weeks known as shaping. The unaffected arm is constrained with a mitt to promote the use (forced use) of the more impaired limb during $90 \%$ of the waking hours [17]. The pooled meta-analysis of 36 trials by Etoom et al (2016) found a significant effect of CIMT on upper extremity function with no significant effect at different durations of follow-up [18]. The aim of a very recent article on CIMT with neurotrophic growth factor upregulation reviewed some of the CNS biomarkers after stroke and their correlations with motor function outcomes in both humans and animals [19]. This review focused on 8 studies on acute and chronic stroke and proved to have increased brain metabolism and cerebral blood flow, secretion of GAP-23, HIF-1a, VEGF and other neurotrophins [20]. We also administered CIMT with neuromuscular electrical stimulation (NMES) and rTMS (repetitive transcranial magnetic stimulation) in chronic stroke subjects and observed short term benefits of CIMT resulting in gains in hand function and MEP pre and post therapy [21].

CIMT \& neural correlates: CIMT is a specialized taskoriented training approach. Its specific strategy is to induce motor learning (practice specificity, feedback) and neuroplasticity (practice-induced brain changes arising from repetition, increasing movement complexity, motivation and reward) with intensive blocks of training. Modified CIMT protocols have been described with dosage regimens ranging from 0.5 to $6 \mathrm{~h}$ per day. Functional neuroimaging studies suggest that increased activity in the ipsilesional sensorimotor and primary motor cortex plays a role in the improvement of functional outcome after task-specific rehabilitation.

\section{(ii) Mirror therapy (MT)/Mental virtual imagery/Action Observation Therapy (AOT)}

A new interventional strategy, named action observation training (AOT) or mirror therapy,was proposed for upper limb motor rehabilitation after stroke, which involves action observation and action execution sequentially. In AOT, participants observe the actions presented via videos or performed by other people.The subjects then repetitively stimulate and practice the observed actions [22].

Tsai-yu Shih et al worked on a study to check the effect of AOT and mirror therapy after stroke, an estimated total of 90 patients with subacute stroke received AOT, MT, or control intervention for a 3-week training period. The results provided scientific evidence of treatment effects and neural activity changes after AOT \& MT and concluded that both the treatments are useful for modern neuro-rehabilitation [23].

A very recent review conducted on 3781 mirror therapy studies by Pandian et al 2019 showed an effective and feasible approach to rehabilitate post-stroke survivors in the acute, sub-acute, and chronic phases of stroke for definitive motor and sensory improvements [24]. One of our study included 20 patients with chronic stroke and 10 healthy controls for webcam based mirror therapy for 8 weeks. Patients had clinical examination, severity scores, functional MRI (fMRI)\&diffusion tensor imaging (DTI) at baseline, 8 and at 24 weeks. All the 
patients showed statistical significant improvement in Fugl Meyer and modified Barthel Index ( $p=0.05)$, also an increase in activation cluster of ipsilesional BA4, BA6 and inferior parietal lobule (BA 40) suggesting MNS activation. This innovation using webcam was patented in 2014 (Patent no; 1781/DEL/2014) [25].

Mirror therapy and neural underpinnings: Mirror therapy utilizes priming mechansim of learning through the ipsilesional brain motor pathway and activates the mirror neuron system (MNS) $[8,26]$. Based on findingsin the academic literature and clinical expertise, (a) active range of motion (AROM) exercises, (b) reaching movement or object manipulation, (c) UE functional tasks can be practiced. Phase 1, the patients observes AROM exercises through video clips, phase 2 the patients observes tasks, depending on the patient's motor ability, through a video clip. There is moderate-quality evidence that mirror therapy is superior to sham therapy, control therapy (task-oriented training, bimanual exercises, symmetric training) or standard rehabilitation with regards to upper extremity impairments and disabilities. Effects of mirror therapy may persist till 6 months after treatment. Mental imagery on the contrary works on the principle of explicit learning. According to Schimdt, the desired motor program is activated by imagery leading to functional reorganisation in unaffected and affected hemispheres $[7,8]$.

\section{(iii) Virtual reality/Haptics in stroke rehabilitation}

Virtual reality (VR) is a technological advancement which allows the users the experience of being surrounded by a computer-generated world which makes them interact as in a real environment [27]. A review by Laver et al included 35 new studies with small study sample sizes and varied interventions in terms of treatment and the devices used [28]. Results were not statistically significant for upper limb function $(95 \% \mathrm{Cl}$ -0.05 to 0.20 ) when comparing virtual reality to conventional therapy. However, when virtual reality was used in addition to usual care (providing a higher dose of therapy for those in the intervention group) there was a statistically significant difference between groups (SMD 0.49, 0.21 to 0.77, 10 studies, 210 participants, low-quality evidence).Twenty-three studies reported that they monitored for adverse events and some studies relatively mild side effects [29].

We designed a VR based haptic-enabled Physiologically Aided (PA) Rehabilitation System for patients with upper limb movement disorders. The VR environment was augmented with tactile feedback by using haptic device (from Geomagic Inc.) with a pen type stylus that can be moved in 3 directions. This novel, multimodal and performance-sensitive exercise platform was experimented on 10 chronic stroke ( $>6$ months) patients on unilateral shoulder abduction and adduction that are essential for the performance of daily living activities. The device resulted in greater accuracy and precision in hand activities and shoulder movements in stroke $(p<0.05)[30,31]$.

Robotics is an upcoming field of neurorehabilitation in stroke. It can be categorized as passive, active and activeassist. The passive tool conducts the movement support regardless of the user's voluntary effort resulting in repeated stretching [32]. The active device needs the voluntary effort of the user to activate movement assistance activation. Pie et. al carried out a survey of 233 physical therapists with suggested features such as the capability to adapt to the hand's movement, the ability to be used while in a seat, feedback to the user, the focus on practicing activities of daily living, the ability to operate at home [33]. Robot rehabilitation showed positive findings and results of the study were very promising. The challenge remains, however, to convert complicated and complex protocols into simple, low cost, customizable and user-friendly devices that can be used even in house settings.

Motor relearning and haptics:

The literature on VR highlights its motor relearning principles through repetition in an enriched environment, confidence through reinforcement and immediate feedback, and positivity through social interaction. Any gains that are obtained are specific to the task that is being trained (motor impairment) and do not extrapolate to upper extremity disabilities in daily life [34].

(iv) Task oriented training/repetitive arm training [35,36] According to the review analysis, a total of 15 sessions of taskoriented training ( 5 times per week for 3 weeks) focussing on arm and hand function with a licensed physical therapist resulted in efficient arm and hand function post stroke. Task-oriented arm training involves the repetitive practice of goal-directed, functional movements. Tasks focuses on proximal arm control (i.e., shoulder/elbow movement), hand grasp (gross grasp, fine motor), object manipulation (tool use, movement of objects within the hand), or the combination of proximal control and hand grasp/object manipulation. Trainings are designedto be individualized, challenging, and progressive in nature.The difficulty level of the motor training was progressed across sessions through changes in task setup (e.g., moving from reaching at midline to reaching across midline), task demands (e.g., increase weight of object lifted), and the task itself (e.g., reach and pick up a cup with a gross grasp to reach and pick up a coin with a pincer grasp while holding additional coins in the same hand).

TOT \& neural dependence: Repetitive arm training leads to implicit and explicit learning in the motor cortex. There is bilateral activation of cerebral cortices followed by brain stem and cerebellar networks [37]. Studies on practice scheduling in motor learning area have been developed based on the assumption that random practice facilitates the transfer and retention of motor skills by adding parameters (e.g. schema enrichment) or strengthening of representations (e.g action plan) of practiced skills in the memory.

\section{Integration of Therapeutic Approaches}

There has been good data on combination therapy being 
Bhasin et al., Physical Therapy and Rehabilitation 2021,

used for stroke functional and motor recovery. Some of the modules adopted are:

a) Cognitive-motor interference: Cognitive-motor interference (CMI) occurs when simultaneous (dual-task)performance of acognitive and a motor task results in deterioration of performance in one or both tasks, relative to performance of each task separately. Thepattern of CMI depends on several factors like types of tasks; levels ofdifficulty, instructions regarding which, if any, task to prioritize; and the characteristics ofthe person performing the task (e.g., cognitive and motor abilities, fear of falling) [38]. Jody A. Feld observed the patterns of cognitive-motor dual-task interference on stroke patients and concluded that dual-task declines in gait speed were highly prevalent at the time of hospitaldischarge and were often associated with reciprocal dual-task declines in the cognitive task,suggesting widespread loss of gait automaticity [39].

b) Motor-sensory integration: Clinical evidence has confirmed that combining sensorimotor training modality is more effective than conventional motor-orientedapproaches. Clinical rehabilitation technologies based on sensory input are Bobath technique, proprioceptive neuromuscular facilitation (PNF) approach, Roods technique, cognitive-motor training, and music-based intervention,during gait training, rhythmic sound stimulation can significantly improve a patient's walking function, especially in terms of posture control [40]. Altenmüller et al have administered a music-based intervention including self-paced movements of the index finger and of the whole arm and they found that the music-supported therapy yielded significant improvement in both gross and fine motor functions of the hands [41].

c) Pharmacological Treatment and Rehabilitation: Dopaminergic agents and selective serotonin-reuptakeinhibitors (SSRIs) are known in the altering of natural historyof recovery after stroke. These compounds have been studied in preclinical and clinical trialsand revealed that SSRIs coupled with physiotherapy aid in functional recovery via modulation of noradrenergic system by exerting an effect on excitation/ inhibition pathways, leading to enhancement of motor function after a stroke [42]. They promote neuronal sprouting and cortical reorganization restoring blood flow thereby improving neuronal survival. This compound regulates the expression of hypoxia-inducible factor-1a (HIF-1a) and of heme oxygense- 1 thereby leading to neuroplasticity and recovery in Stroke.

The FLAME trial was conducted in nine centres in France, in 118 patients withischemic stroke and unilateral weakness. Treatment with fluoxetine, started between days 5 and 10 aftersymptom onset, improved motor recovery and increasedthe chances of functional independence after 3 months [43]. FOCUS was a pragmatic, randomised, placebo-controlled trial that recruited 3127 adult patients with ischemic stroke or intracerebral haemorrhageacross 103 hospitals in the UK, over a period of 4.5 years. Patients were randomly allocated fluoxetine $20 \mathrm{mg}$ once daily or placebo, initiated between
2 days and 15 days after stroke onset andcontinued for 6 months. Unfortunately, the trial did not demonstrate any benefit on functional outcome of fluoxetine compared with placebo at 6 months (OR 0.951 [95\% Cl 0.839-1.079]; $p=0.439$ ) [44]. We attempted a combination of fluoxetine and tDCS in chronic stroke to determine the effectiveness of combination therapyfor postural stability and gait using fluoxetine with tDCS and DTT. The results of the same study are awaited [45].

Non invasive brain stimulation (NIBS) in stroke $[46,47]$ The amalgamation of bioelectrical and engineering domains with medical sciences has led to the development of newer technologies. The stimulation through TMS or TDCS induces behavioral changes and the response of the brain is captured/ modulated or augmented by these modalities. NIBS for stroke patients works on two theoretical models as suggested by reviewers: (i) an interhemispheric inhibition of human motor cortices on one another; and (ii) the transcallosal inhibitory effect on the affected motor cortex because of the above said phenomenon. The approach for applying these gadgets is to either up-regulate the lesional hemisphere with excitatory anodal stimulation or down-regulate the contralesional hemisphere with inhibitory cathodal stimulation.

\section{fMRI \& Stroke recovery}

Based on information gained from both short-term and long-term assessment of recovery following stroke, it may be possible to define two distinct phases of recovery in brain activity-recruitment and focusing. The recruitment phase increases the population of available neurons to compensate for lost connections, particularly supplementary cortical regions and contralesional regions. FMRI and DTI have been a boon to understand the functional and structural recovery post stroke. The experience and environment rich plasticity has been well elucidated in research trials $[\mathbf{8 , 9 , 3 1 ]}$. In another interesting research, we conducted $\mathrm{fMRI}$ analysis trial on functional imaging and effects of physiotherapy on diffusion tensor imaging at baseline, pre and post physiotherapy (8 weeks). Multivariate regression analysis at baseline showed that rFA well correlated to the Fugl-Meyer score (regression coefficient:0.198, $\mathrm{F}=10.39, \mathrm{p}=0.001$ ) and motoricity index (MI). All patients had high \% signal intensity after 8 weeks of physiotherapy regime with a greater percentage change in rFA as compared at follow up suggesting that a focused exercise regime in stroke patients helps in the reconnection of neural and myelin networks [49].

\section{Conclusion}

We attempted to synthesize some of the effective therapeutic exercise regimes which are practiced widely to promote neural recovery post stroke. These 'ingredients of cognitive neuroscience' for motor recovery have been evidenced by our research experience as well [50]. We hope that future work in motor recovery after stroke will encompass a more structured and 
Bhasin et al., Physical Therapy and Rehabilitation 2021,

novel rehabilitation protocols along with other biosurrogate markers in stroke and other patient populations.

\section{Competing interests}

The authors declare that they have no competing interests.

\section{Authors' contributions}

\begin{tabular}{|l|c|c|c|c|}
\hline Authors' contributions & AB & NK & MVPS & SK \\
\hline Research concept and design & $\sqrt{ }$ & $\sqrt{ }$ & -- & -- \\
\hline Collection and/or assembly of data & -- & $\sqrt{ }$ & -- & -- \\
\hline Data analysis and interpretation & $\sqrt{ }$ & -- & -- & -- \\
\hline Writing the article & $\sqrt{ }$ & $\sqrt{ }$ & -- & $\sqrt{ }$ \\
\hline Critical revision of the article & -- & -- & $\sqrt{ }$ & $\sqrt{ }$ \\
\hline Final approval of article & -- & -- & $\sqrt{ }$ & $\sqrt{ }$ \\
\hline Statistical analysis & $\sqrt{ }$ & -- & -- & -- \\
\hline
\end{tabular}

\section{Publication history}

Editor: Catherine Ortega, University of Texas Health Science Center, USA. Received: 26-April-2021 Final Revised: 18-June-2021

Accepted: 23-June-2021 Published: 08-July-2021

\section{References}

1. Masaki H, Sommer W. Cognitive neuroscience of motor learning and motor control. J Phys Fitness Sports Med 2012;1(3):369-380.

2. Darwin, C. On the origin of species by means of natural selection, or the preservation of favoured races in the struggle for life. Book London: 1859; John Murray.

3. Dahms C, Brodoehl S, Wittie OW, Klingner CM. The importance of different learning sequence strategies for motor learning after stroke. Hum Brain Mapp. 2020;41:270-286.

4. Haeckel E. Generellemorphologie der organismen (Vols.1-2). 1866;Berlin: G. Reimer.

5. Alia C, Cristina Spalletti, Stefano Lai, Alessandro Panarese, Giuseppe Lamola, Bertolucci F, Fabio Vallone, Angelo Di Garbo, Carmelo Chisari, SilvestroMicera and MatteoCaleo. Neuroplastic Changes Following Brain Ischemia and their Contribution to Stroke Recovery: Novel Approaches in Neurorehabilitation. Frontiers in Cellular Neuroscience 2017; Volume 11;Article 76.

6. Schmidt RA. Motor schema theory after 27 years: reflections and implications for a new theory. Research quarterly for exercise and sport. 2003;74(4):366-375.

7. Shea CH, Wulf G. Schema Theory: A Critical Appraisal and Reevaluation. Journal of Motor Behaviour 2005;37(2):87-101.

8. Newell K, Verhoeven FM. Movement rehabilitation: are the principles of re-learning in the recovery of function the same as those of original learning? Disability and rehabilitation 2016;39(2):1-6.

9. Maier M, Ballester, BR, Verschure PJ. Principles of neurorehabilitation after stroke based on motor learning and brain plasticity mechanisms. Frontiers in Systems Neuroscience 2019;13:74-81.

10. Kleim JA. Neural plasticity and neurorehabilitation: Teaching the new brain old tricks. Journal of Communication Disorders 2011; 44(5), 521-528.

11. Boyd LA, Vidoni E, Wessel BD. Motor learning after stroke: Is skill acquisition a prerequisite for contralesionalneuroplastic change? Neuroscience letters 2010;482(1):22-27.

12. Seidler RD, Bo J, Anguera JA. Neurocognitive contributions to motor skill learning. The role of working memory. J of Mot Behav 2012;44(6):445453.

13. Kal E, Winters M, Van Der Kamp J, Houdijk H, Groet E, Van Bennekom $C$, et al. Is Implicit Motor Learning Preserved after Stroke? A Systematic Review with Meta- Analysis. 2016;1-23.

14. Censor N, Dimyan MA, Cohen LG. Modification of existing human motor memories is enabled by primary cortical processing during memory reactivation. Curr Biol. 2010;20(17):1545-9.

15. Winstein C, Lewthwaite R, Blanton SR, Wolf LB, Wishart L. Infusing Motor Learning Research Into Neurorehabilitation Practice: A Historical Perspective With Case Exemplar From the Accelerated Skill Acquisition Program. J NeurolPhysTher. 2014 Jul;38(3):190-200.

16. Srivastava MP, Kuthiala N. Post Stroke Restorative Therapies: Drugs, Devices and Robotics. Astrocyte 2018; 5:39-52.

17. Kwakkel G, Veerbeek JM, Wegen EH, Wolf SL. Constraint-induced movement therapy after stroke. The Lancet Neurology 2015;14(2):224234.

18. Etoom M, Hawamdeh M, Hawamdeh Z, Alwardat M, Giordani L, Bacciu S, Scarpini C, Foti G. Constraint-induced movement therapy as a rehabilitation intervention for upper extremity in stroke patients: systematic review and meta-analysis. Int J of Rehab Research 2016;39(3):197-210.

19. Abdullahi A, Truijen S, Saeys W. Neurobiology of Recovery of Motor Function after Stroke: The Central Nervous System Biomarker Effects of Constraint-Induced Movement Therapy. Neural Plasticity 2020;15:9484298.

20. Venkat A, Taimni V, Bhasin A, Srivastava MV. Comparison of CIMT and NMES in chronic stroke measured on disability functional scales. Indian Journal of Physical and Occupational Therapy 2015;9(2):182-187.

21. Kuthiala N, Bhasin A, Sharma R, Srivastava MV,SKumran S, Sharma S, Kumar N. rTMS and CIMT for Neurofunctional Recovery in Chronic Stroke. Int J NeurorehabilitationEng; 2020:Volume7:6.DOI: 10.37421/ ijn.2020.7.378.

22. Kuk EJ, Kim JM, Oh DW, Hwang HJ. Effects of action observation therapy on hand dexterity and EEG based cortical activation patterns in patients with post-stroke hemiparesis. Top Stroke Rehabil. 2016;23(5):318-25.

23. Tsy S, Wu CY, Lin KC, Cheng CH, Hsieh YW, Chen CL, Lai CJ, Chen CC. Effects of action observation therapy and mirror therapy after stroke on rehabilitation outcomes and neural mechanisms by MEG: study protocol for a randomized controlled trial. Trials 2017;1-464.8:459.

24. Gandhi DBC, Sterba A, Khatter H, Pandian J. Review: Mirror Therapy in Stroke Rehabilitation: Current Perspectives. Therapeutics and clinical risk management 2020:16;75-85.

25. Bhasin A, Srivastava MV, Bhatia R, S Mohanty S. Neural interface of mirror therapy in chronic stroke: a functional imaging study. Neurology India 2012; 60(12):570-576.

26. Stoykov ME, Corcos DM, Madhavan S. Movement-Based Priming: Clinical Applications and Neural Mechanisms. J OF Motor behavior 2017; 49(1):88-97.

27. Laver K, George S, Thomas S, Deutche JE, Crotty M. Virtual reality for stroke rehabilitation: an abridged version of a Cochrane review. Eur J PhysRehabil Med 2015;51(4):497-506.

28. Laver K, George S, Thomas S, Deutche JE, Crotty M Cochrane review: virtual reality for stroke rehabilitation. Eur J PhysRehabil Med. 2012;48(3):523-30.

29. Piggot L, Wagner L, Ziat M. Haptic Neurorehabilitation and Virtual Reality for Upper Limb Paralysis: A Review. Critical Reviews in Biomedical Engineering 2016;44: 1-32.

30. Dhiman A, Solanki D, Bhasin A, Bhise A, Das A, Lahiri U. Design of adaptive haptic enabled virtual reality based system for upper limb movement disorders. A usability study. Proceedings of IEEE RASE EBMS 2016; 4: 1254-1259.

31. Dhiman A, Solanki D, Bhasin A, Das A, Lahiri U. An intelligent, adaptive, performance-sensitive, and virtual reality-based gaming platform for the upper limb. ComputAnim Virtual Worlds. 2018;e1800;1-14.

32. Pei Y-C, Chen J-L, Wong AMK, Tseng KC. An Evaluation of the Design and Usability of a Novel Robotic Bilateral Arm Rehabilitation Device for Patients with Stroke. Front Neurorobot 2017;11:36-42.

33. Macclellan LR, Bradham DD, Whitall J, Volpe B, Wilson PD, Ohlhoff J, et al. Robotic upper-limb neurorehabilitation in chronic stroke patients. J Rehabil Res Dev . [cited 2019 Jul 22];42(6):717-22.

34. Takahashi CD, Der-Yeghiaian L, Le V, Motiwala RR, Cramer SC. Robot- 
Bhasin et al., Physical Therapy and Rehabilitation 2021,

based hand motor therapy after stroke. Brain. 2008;131(2):425-37.

35. Lang CE, Birkenmeier RL. Upper-Extremity Task Specific Training after Stroke or Disability: A Manual for Occupational Therapy and Physical Therapy, The American Occupational Therapy Association, Inc., Bethesda, MD, 2014.

36. Hatem SM, Saussez G, Bleyenheuft Y. Rehabilitation of Motor Function after Stroke: A Multiple Systematic Review Focused on Techniques to Stimulate Upper Extremity Recovery. Front Human neuroscience 2017;10:442-448.

37. Stewart JC, Handlery K, Jessica F. Baird, Blanck EL, Pathak G, and Stacy L. Fritz. Targeted Engagement of the Action Selection Network during TaskOriented Arm Training after Stroke. Neural Plasticity 2020;vol:2:Article ID 8814158.

38. Prudence Plummer, Gail Eskes, Sarah Wallace, Clare Giuffrida, Michael Fraas, Grace Campbell, Kerry Lee Clifton, Elizabeth R. Skidmore, and on behalf of the American Congress of Rehabilitation Medicine Stroke Networking Group Cognition Task Force. Cognitive-Motor Interference during Functional Mobility after Stroke: State of the Science and Implications for Future Research Arch Phys Med Rehabil. Arch Phys Med Rehabil. 2013; 94(12):112-117.

39. Jody A Feld Patterns of cognitive-motor dual-task interference poststroke: an observational inpatient study at hospital discharge Prudence PLUMMER, European Journal of Physical and Rehabilitation Medicine 2020;16:DOI: 10.23736/S1973-9087.20.06273-5.

40. Xiaowei Chen, Fuqian Liu, Zhaohong Yan, Shihuan Cheng, Xunchan Liu, $\mathrm{He} \mathrm{Li}$, Zhenlan Li. Therapeutic effects of sensory input training on motor function rehabilitation after stroke. Medicine 2018;97:48-53.

41. Altenmüller E, Marco-PallaresJ,Münte TF et al. Neural reorganization underlies improvement in stroke-induced motor dysfunction by musicsupported therapy. Ann New York AcadSci 2009;1169:395-405.

42. Srivastava MV, Bhasin A, Talwar T, Moonis M. Pharmacological Agents in Post Stroke Recovery. J Neurol Stroke 2014;1(6):00040.DOI: 10.15406/ jnsk.2014.01.00040

43. Chollet F, Tardy J, Albucher JF, et al. Fluoxetine for motor recovery after acute ischemic stroke (FLAME): a randomized placebo-controlled trial. Lancet Neurol 2011;10:123-30.

44. FOCUS Trial Collaboration. Effects of fluoxetine on functional outcomes after acute stroke (FOCUS): a pragmatic, double-blind, randomized, controlled trial. Lancet 2018.

45. Roy B, Srivastava MVP, Bhatia R, Kumar N, Wadhwa S. Effect of DualTask Exercise in conjunction with Fluoxetine \&Transcranial Direct Current Stimulation on Postural Stability and Gait in Stroke Patients. Neurorehabilitation and Neural Repair 2018; Vol 32:Issue 4-5.

46. Williams JA, Imamura M, Fregni F. Updates on the use of non-invasive brain stimulation in physical and rehabilitation medicine. J Rehabil Med 2009; 41:305-311.

47. Fregni F, Pascual-Leone A. Technology Insight: noninvasive brain stimulation in neurology-perspectives on the therapeutic potential of rTMS and tDCS. Nature. ClinPractNeurol 2007;3(7):383-93.

48. Crofts A, Kelly ME, Gibson CL. Imaging Functional Recovery Following Ischemic Stroke: Clinical and Preclinical fMRI Studies. J of Neuroimaging 2020;30(1):5-14.

49. Bhasin A, Srivastava MVP, Kumaran S. Correlation of DTI derived measures to therapy mediated recovery after Stroke. Preliminary Findings. Neurology India 2020 (ahead of print).

50. Weinstein CJ, Kay DB. Translating the science into practice: shaping rehabilitation practice to enhance recovery after brain damage. Prog Brain Res 2015:218:331-60.

\section{Citation:}

Bhasin A, Kuthiala N, Srivastava MVP and Kumaran S. Neural substrates of Motor Learning Strategies in Stroke. Phys Ther Rehabil. 2021; 8:4.

http://dx.doi.org/10.7243/2055-2386-8-4 